\title{
Female Students Knowledge of Breast Self-examination in a University in the Western Cape
}

\author{
Mavis Bobie Ansah" ${ }^{1 *}$ and Hilda Vember ${ }^{2}$ \\ ${ }^{1}$ Komfo Anokye Teaching Hospital, Kumasi-Ghana \\ ${ }^{2}$ Cape Peninsula University of Technology, Symphony Way Bellville, Cape Town, South Africa
}

\begin{abstract}
The most common cancer in women worldwide is breast cancer. It is also the leading cancer affecting women in South Africa. When breast cancer is detected early, it improves the outcome of the disease and reduces mortality. The knowledge of female university students were assessed by the use of self-administered questionnaires and oneon-one interview. Participants lacked knowledge on breast cancer risk factors, as majority of them only knew about family history being a risk factor. Majority of the participants had never been educated by their healthcare provider on breast cancer and its screening.
\end{abstract}

Keywords: Breast cancer; Breast cancer screening; Female; University students; Breast self-examination; Cape Town

\section{Introduction}

The most common cancer in women worldwide is breast cancer. It has become a major public health issue throughout the world. Of the new breast cancer diagnosis, five per cent (5\%) occurs in women who are less than 40 years of age. When compared to women who are 40 and older, the 5-year relative survival of young women who are 40 or less is at a slighter lower rate. It is critical for young women to screen for early detection and to have knowledge about risk reduction. According to the National Cancer registry, breast cancer is the leading cancer in South African women with a life time risk of 1:35. South African women often present to the hospital with advance cancer. This occurs especially among black women. The cancer related health seeking behaviors of South African women are influenced by cultural beliefs such as witchcraft, mistrust of medical services and the risk factors of cancer, such as smoking and alcoholism. There is a racial variation in the ages at which South African women present with breast cancer. Colored, Indian and Black women are diagnosed at a younger age than their white counterparts. However, Black women report to the hospital at advance stages of the disease, Colored's at a stage considered being intermediate and whites report at an early stage.

When breast cancer is detected early, it improves the outcome of the disease and reduces mortality [1]. Breast cancer screening involves the use of a mammogram, clinical breast examination and breast selfexamination. Breast self-examination (BSE) is a painless and free method of breast cancer screening which can easily be practiced. BSE is recommended as an approach which can be used to increase the breast cancer awareness. BSE has the potential for allowing early detection of any abnormalities of the breast [2].

\section{Materials and Methods}

\section{Sample, data collection method and research design}

The target population for this study was registered female students who were staying on one of the residences of the selected campus of the university under study. The total number of females staying on the chosen campus for the study is nine hundred and ten (910). The sample included only female university students who were staying on the residences where the study was taking place. For this study, 207 participants were recruited to complete the questionnaire and 12 participants were recruited to be interviewed [3]. The sample size was determined by the use of Raosoft sample size calculator online software. The confidence level used was $94 \%$, with a margin of error of $6 \%$ and response distribution of 50\%. Sharma endorses the use Raosoft sample size calculator in nursing research. The sample for this study was selected by the use of convenience sampling. The researcher went to the residences from Monday to Friday, during the hours of 9:00 h to 16:00 h. Female students, who were available at the time of data collection and readily agreed to participate in the study, were included in the study. Two hundred (207) questionnaires were distributed. However, only one hundred and fifteen $(n=115)$ participants responded. Twelve participants were interviewed $(n=12)$. In total one hundred and twenty seven students participated in this study $(\mathrm{N}=127)$ [4].

We used a descriptive mixed method design to assess the knowledge of BSE among female university students. The necessary data were collected from female students who were residents on one campus of the selected university for the study. Two data collection tools were used in this study [5]. A self-administered questionnaire was used to collect quantitative data, while one-on-one interviews were used to collect qualitative data. The questionnaire was divided into five sections in an alphabetical order. Section A was to collect data on the participants' demographic characteristics; section B was meant to collect data on participants' knowledge of breast cancer and BSE. In all, there were 31 questions in the questionnaire. A face-to-face interview, also known as one-on-one was used for this study. During the interview, any misunderstood questions were clarified by the researcher [6]. There was a moderator present at the interviews. The participants' level of understanding of BSE was assessed using interviews. Also, a more rich and complex data pertaining to the knowledge, beliefs, attitude and practice of BSE among female university students was collected.

*Corresponding author: Mavis Bobie Ansah, Komfo Anokye Teaching Hospital, Kumasi-Ghana, Tel: 0021671577850; Fax: 0021671574725; E-mail: nyamekyem@gmail.com

Received January 25, 2018; Accepted February 15, 2018; Published February 22, 2018

Citation: Ansah MB, Vember H (2018) Female Students Knowledge of Breast Selfexamination in a University in the Western Cape. J Oncol Med \& Pract 1: 115 doi:10.4172/2576-3857.1000115

Copyright: () 2018 Ansah MB, et al. This is an open-access article distributed under the terms of the Creative Commons Attribution License, which permits unrestricted use, distribution, and reproduction in any medium, provided the original author and source are credited. 
In all there were thirteen questions that were used to assess the participant's knowledge on breast cancer and breast cancer screening.

For demographic characteristics, age, marital status, religion and residence were items that were reported. For the sake of analysis, age was grouped into three groups as 16-25, 26-30 and 31-35 years. Marital status was measured with four categories, single, married, divorced and separated. Since South Africa has a lot of religion being practiced, religious beliefs were measured with five categories, thus, Christian, Muslim, Traditional beliefs, Hindu and other. The participants were asked to mention the residence in which they stayed. The study was carried out in 9 out of the 10 residences on campus; the reason being that 1 of the residence is a male's residence [7].

\section{Quantitative data analysis}

Responses from questions were captured and analyzed by the use of Statistical package for the Social Science (SPSS), 2015 and Microsoft Excel 2010. Each question was captured separately from all respondents example all question 1 responses were captured onto one Excel spread sheet. Similarly all question 2 responses were treated in the same manner. The data was then examined for frequency distribution of responses using SPSS. Elements of descriptive statistics, such as frequency distribution and percentages of responses on were analyzed [8].

\section{Qualitative data analysis}

All the interviews were conducted by the researcher in the presence of a moderator. The interviews were transcribed into a Microsoft word 2010 document within 24 hours after they were conducted. Transcriptions began immediately after the first interview and ended with the transcription of the last interview (Davis, 2006: 41). The researcher then listened to the audio responses again and compared them to the typed document. The transcribed data was given back to respondents to verify if that was the information they gave. Responses were coded and grouped under different themes as they emerged. Responses on each theme were then analyzed using thematic analysis $[9,10]$.

\section{Results and Discussion}

\section{Quantitative data}

Demographic data: Participants were between 18 years and 35 years. Participants within the ages of $18-25$ were $(n=103,89.57 \%)$, participants within the ages of $26-30(n=10,8.69 \%)$ and those within the ages of 31-35 were $(n=2,1.74 \%)$. Both postgraduate and undergraduate students participated in this research. One hundred and fourteen of participants were single $(n=114,99.13 \%)$ and only one $(n=1,0.87 \%)$ was married. None of the participants were separated or divorced. The attitudes, beliefs and knowledge of participants have no significant relation with their marital status. One hundred and eight participants ( $n=108,95.58 \%)$ were Christians, there were no Muslims among the participants, two participants $(\mathrm{n}=2,1.77)$ were traditional believers, one Hindu $(n=1,0.88 \%)$ and two had other believes $(n=2,1.77)$ which were not mentioned in the questionnaire. Although there is dominance in Christianity in South Africa, there is a strong influence of African traditional religion which is unquestionable. Majority of participants were Christians.

Participants were asked to name their residence; this was to make sure participants met the inclusion criteria. There were 15 participants from residence $1(n=15,13.04)$, ten participants stayed in residence 2 $(n=10,8.7 \%)$, and residence 3 had twelve participants $(n=12,10.43 \%)$.
From residence 4, fifteen participants $(n=15,13.04)$, there were four participants from residence $5(n=4,3.48 \%)$ and residence 6 had fifteen participants $(n=15,13.04)$. Residence 7 had eight participants $(n=8$, 6.97), twelve students participated from Residence $8(n=12,10.43 \%)$ and the highest number of participants which is twenty four, stayed at Residence $9(n=24,20.87 \%)$ [11].

\section{Knowledge}

Heard about breast cancer: One hundred and twelve participants had heard of breast cancer before $(n=112,97.39 \%)$. Three participants had never heard of breast cancer before $(n=3,2.61 \%)$. This can be seen in chart below. One of the conditions that must be fulfilled in order to get knowledge is a belief or approval condition, this is according to the traditional conception of knowledge, also known as epistemology. As shown by this result, majority of the participants had knowledge of breast cancer being an existing disease (Chart 1).

Knows anyone that has had breast cancer: One hundred and fourteen participants responded to this question $(\mathrm{N}=114)$ and one did not. Of the one hundred and fourteen, forty six knew somebody who has had breast cancer $(n=46,40.35 \%)$, whereas sixty eight did not know anyone with breast cancer $(n=68,59.65 \%)$. According to the traditional conception of knowledge, another condition that must be fulfilled in order to gain knowledge, is a truth condition, thus, getting to know that other women has had breast cancer. The result shown in Chart 2 indicates that most of the participants did not know anyone who has had breast cancer before.

The gender which breast cancer affects: As indicated in Chart 3 below, no participant answered that men only had breast cancer $(n=0)$. Fifty nine participants answered that breast cancer affects women only $(n=59,51.30 \%)$, forty eight participants answered that breast cancer affects both men and women $(\mathrm{n}=48,41.74 \%)$ and eight participants did not know $(n=8,6.97 \%)$. Breast cancer is the most common female cancer in the world and is the leading cause of cancer death in women. Breast cancer rarely occurs in men, with less than $1 \%$ of all breast cancers occurring in men. The results shown in Chart 3 confirm that breast cancer mostly occurs in women as most participants were not aware of male breast cancer [12]. Participants were asked to tick as many risk factors of breast cancer they knew. One hundred and seven participants responded to this question $(\mathrm{N}=107)$ and eight did not respond.

\section{Risk factors}

Family history: Of the one hundred and seven, one hundred and two indicated that family history is a risk factor of breast cancer $(\mathrm{n}=102,95.33 \%$ of 107$)$. Family history is a key risk factor for breast cancer. Women who have a first-degree relative (i.e. mother or sister) with breast cancer have a 1.7-2.5 risk for developing breast cancer and women with second-degree relatives (i.e. grandmother or

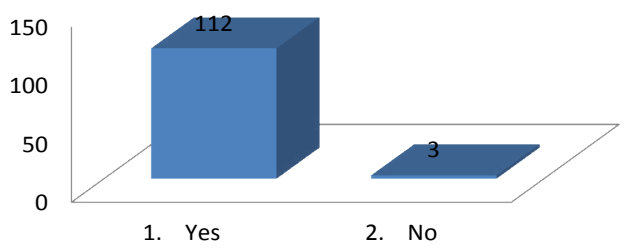

Chart 1: Heard about breast cancer. 


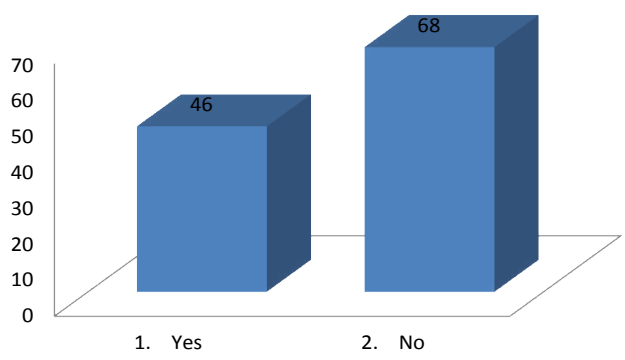

Chart 2: Knows anyone with breast cancer.

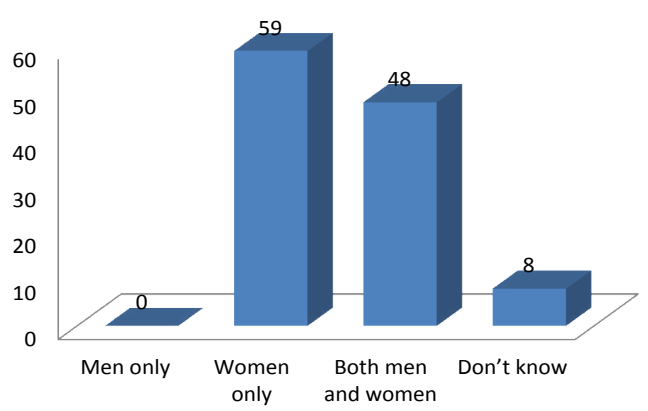

Chart 3: The gender which breast cancer affects.

aunt) with breast cancer, have a 1.5 risk of developing breast cancer. Zhou conducted a study to determine the family history and ductal carcinoma in situ and triple negative breast cancer risk among Han Chinese women in China, it was revealed that, participants who had a strong family history of breast cancer, had a higher risk of getting breast cancer than those who did not have any family history. Majority of the participants had knowledge of family history being a risk factor for breast cancer.

Excessive alcohol intake: There were twenty three indications that excessive alcohol intake, is a risk factor ( $n=23,21.50 \%$ of 107). Park maintains that, alcohol is an established risk factor of breast cancer. In epidemiological studies, it was proved that alcohol consumption at higher levels and to an extent, moderate level, has been linked to increased breast cancer risk. A study conducted in Uruguay by Ronco \& Stefani revealed that, breast cancer risk has a direct association with the number of years of drinking alcohol. Majority of the participants did not know that excessive intake of alcohol increases the chances of getting breast cancer.

Excessive fat intake: Excessive fat intake was indicated to be a risk factor by seventeen participants ( $\mathrm{n}=17,15.89 \%$ of 107). Dietary factors like high-fat diet, beef and high-fat milk products particularly, have been linked to an increase in the risk of breast cancer. Women who have a high total fat intake have a $13 \%$ increased risk of breast cancer. Also, an increased intake of saturated fat increases the risk of getting breast cancer. Majority of the participants did not know that high consumption of fatty foods increases a woman's risk of getting breast cancer. This can be seen in Chart 4 .

Increasing age: There were thirty six indications of increasing age being a risk factor for breast cancer ( $n=36,33.64 \%$ of 107$)$. Breast cancer risk increases with age. Of all breast cancers, $78 \%$ occurs in women who are older than 50 years. $80 \%$ of breast cancer mortality occurs in women who are older than 50 years. Most of the participants did not have any prior knowledge of increasing age being a risk factor for breast cancer.

Reproductive factors (Null parity, early menstruation and late menopause): Not giving birth, was indicated as a risk factor by five responses ( $n=5,4.67 \%$ of 107$)$. There were seven indications that menstruating early is a risk factor for breast cancer $(n=7,6.54 \%$ of 107). There were twelve indications that late menopause is a risk factor ( $n=12,11.2 \%$ of 107). The reproductive history of a woman has been shown as a contribution to breast cancer risk. According to Horn reproductive factors that increase a woman's breast cancer risk are early menarche, late menopause and null parity or low parity. A study carried out in Vermont University in the USA revealed that, there is a strong association of risk of breast cancer in women who have not given birth. The result from Chart 4 indicates that, the majority of the participants did not know that not giving birth, early menarche and late menopause are risk factors of breast cancer [13].

Obesity: There were twenty two responses that obesity is a risk factor of breast cancer $(n=22,20.56 \%$ of 107). There is an increased risk of breast cancer associated with adult weight gain. Obesity affects the development of breast cancer. The percentage of body fat has a positive link with breast cancer risk. As shown in Chart 4, most of the participants did not have prior knowledge that obesity is a risk factor for breast cancer.

\section{Received information from healthcare giver about breast cancer and breast cancer screening}

One hundred and thirteen participants $(\mathrm{N}=113)$ responded to this question. As shown in Chart 5, twenty three participants had received information from their healthcare giver about breast cancer $(n=23,20.35 \%)$. However, ninety participants had never received any information about breast cancer from their healthcare giver $(n=90$, 79.65\%). Yan-qiong and Xiaoyan conducted a study in a community health catchment area in China to determine the effect of breast cancer health education on the knowledge, attitudes and practice behaviors of BSE among women living in that area. It was revealed that the women reported high incidence of self-examination and the proper method of doing BSE were found to be significantly improved after they had been

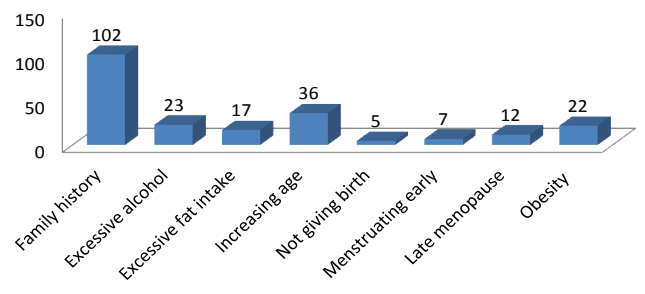

Chart 4: Risk factors of breast cancer

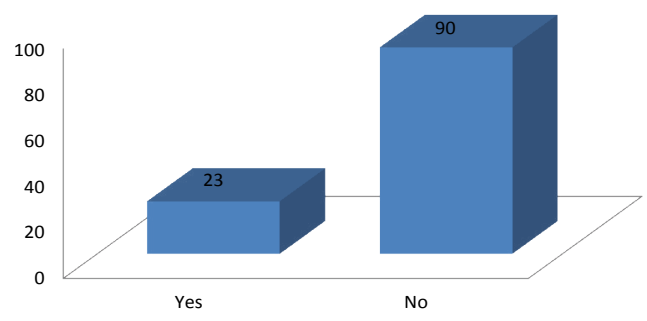

Chart 5: Received information from healthcare giver. 
educated on breast cancer and breast cancer screening. The majority of the participants had never been educated by their healthcare provider on breast cancer and breast cancer screening.

Only fifty five participants answered this question $(\mathrm{N}=55)$. Eleven of the participants gave correct information about breast cancer $(n=11$, $20 \%)$. Ten gave incorrect information about breast cancer $(n=10$, $18.18 \%)$. Thirty four participants gave no information about breast cancer $(\mathrm{n}=34,61.82 \%)$. This can be seen in Chart 6. According to Minelli and Breckon changing habits are involved in health education, but, before habits can be changed, attitudes need to be changed. In order to provide healthcare interventions that are culturally congruent among multicultural groups, the practices of health care and beliefs that are related to them must be explored within these groups by health educators and health care practitioners in general. The findings below show that, majority of participants had not been educated or informed on issues relating to breast cancer.

\section{Knows symptoms of breast cancer}

Only one hundred and eleven participants answered this question $(\mathrm{N}=111)$. As shown in Chart 7 below, eighty participants knew some symptoms of breast cancer $(\mathrm{n}=80,72.1 \%)$ while thirty one did not know any symptoms of breast cancer $(n=31,27.9 \%)$. Over $80 \%$ of women, who participated in a study in a low resourced community in South Africa, were uninformed about warning signs of breast cancer (Brinton et al. 2014: 467-478). In contrast, most of the participants had prior knowledge on the signs and symptoms of breast cancer.

\section{Symptoms of breast cancer}

Participants were asked to tick as many options as applied. This question was answered by one hundred and twelve participants $(\mathrm{N}=112)$. From Chart 4, there were thirty three indications that painless breast mass as a symptom of breast cancer $(n=33,29.46 \%$ of 112). A bloody nipple discharge was indicated as a sign of breast cancer by thirty six respondents $(n=36,32.14 \%$ of 112$)$, twenty eight indicated that tenderness of the breast was a symptom of breast cancer $(n=28$, $25 \%$ of 112 ). There were also thirty participants who pointed out that an ulcer in the breasts was a symptom of breast cancer $(n=30,26.78 \%$ of 112). Swelling of the breast had forty seven suggestions that it is a symptom of breast cancer ( $n=47,41.96 \%$ of 112$)$ and nipple retraction had twenty two responses of being a symptom of breast cancer $(n=22$, $19.64 \%$ of 112). There were fifty suggestions that lump in the armpit is a symptom of breast cancer $(n=50,44.64 \&$ of 112$)$, whereas skin changes in the breast had thirty six indications of being as symptom of breast cancer $(n=36,32.14 \%$ of 112$)$. However, breast pains in the breast before menses which is not a symptom of breast cancer, had seven responses as being a symptom of breast cancer $(n=7,6.25 \%$ of 112$)$. Also, different sizes of breast since childhood which is not a symptom of breast cancer also had seven suggestions as being a sign of breast cancer $(n=7,6.25 \%$ of 112$)$. Six participants responded that they did not have any prior knowledge about symptoms of breast cancer $(n=6$, $5.36 \%$ of 112 ).

Early stage breast cancer usually presents as a slightly tender or painless breast mass. Patients, whose diseases are advanced, may present with breast masses which are palpable, skin and nipple retraction, breast tenderness, nipple discharge that may be bloody or serous in nature. There may be a change in size, texture and shape of the breast. Other clinical features are redness, ulceration, dilated veins or edema, skin changes called "peau derange" and lymph node enlargement in the axilla. The results from question 9 (Chart 8) do not correspond with the findings from question 8 (Chart 7) where eighty

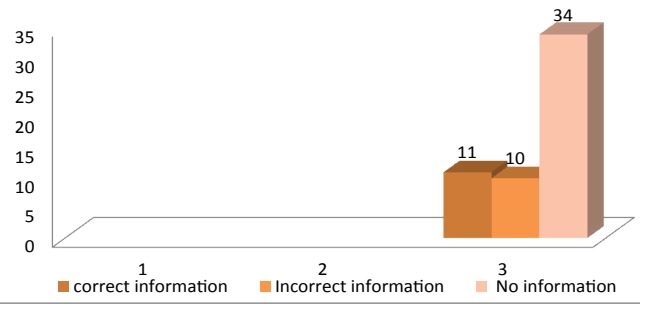

Chart 6: Information received

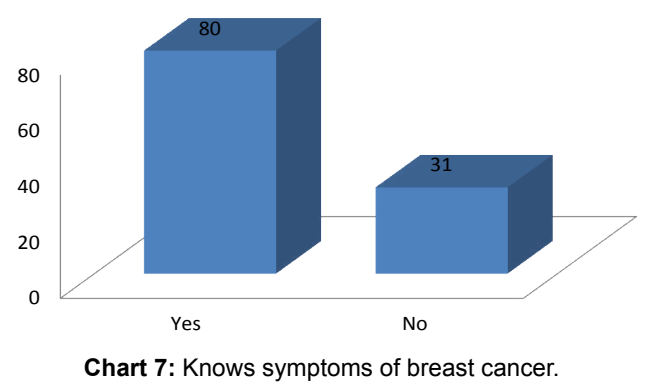

Chart 7: Knows symptoms of breast cancer.

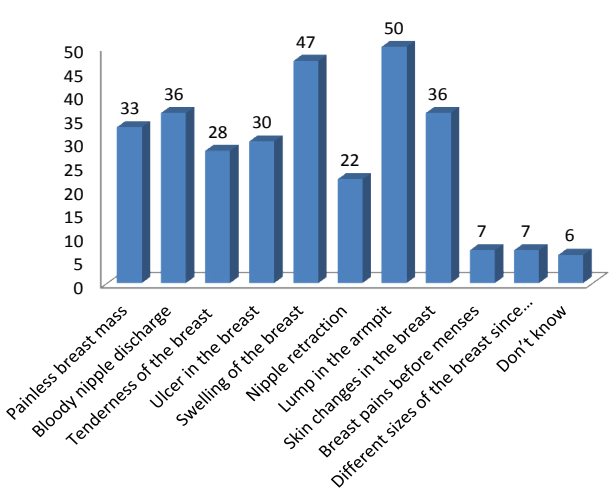

Chart 8: Symptoms of breast cancer.

out of one hundred and one participants had knowledge on signs and symptoms of breast cancer.

Awareness of breast cancer screening: From the chart below (Chart 9), one hundred and fourteen participants responded to this question $(\mathrm{N}=114)$. Sixty four participants were aware of breast cancer screening $(n=64,56.14 \%)$ and the remaining fifty were not aware of breast cancer screening $(\mathrm{n}=50,43.86 \%)$. According to Hoefek an important role can be played by nurses in the promotion of breast cancer screening. The major role of the nurse is to teach screening guidelines and create awareness among women. As shown by the results above, most of the participants were aware of screening for breast cancer.

Heard of breast self-examination: One hundred and fourteen participants responded to this question $(\mathrm{N}=114)$. From Chart 10, eighty one participants had heard of BSE before $(n=81,71.05 \%)$ while thirty three had never heard of BSE before $(n=33,28.95 \%)$. According to Concoran in relation to health, the level of knowledge an individual has is important. Knowledge has an important role to play in the behaviors of health. According to the Information-Motivational-Behaviour model, knowledge in the form of information on health is required for an individual to approve health behaviour. Majority of the participants had prior knowledge on BSE. 


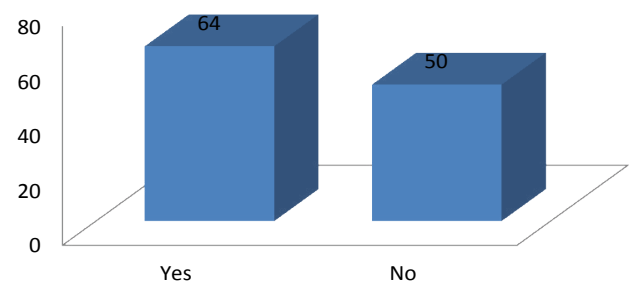

Chart 9: Awareness of breast cancer screening.

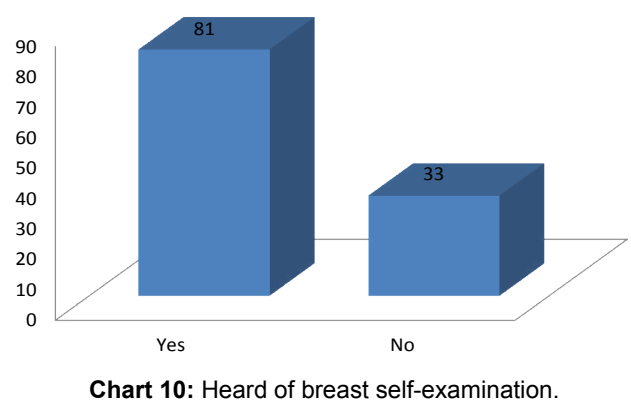

\section{Source of information on breast self-examination}

Participants were asked to tick as many sources of information they have heard about BSE from. In all, eighty nine participants responded to this question $(\mathrm{N}=89)$. The media had sixty three indications as a source of information where participants had heard information on BSE from ( $n=63,70.79 \%$ of 89 ), healthcare professionals had thirty indications as a source of information on BSE ( $n=30,33.70$ of 89$)$. Family members had been a source of information for seventeen participants, $(n=16$, $19.10 \%$ of 89 ) and sixteen participants had heard about BSE from friends ( $n=16,17.98 \%$ of 89 ). David et al. (2010: 174) maintains that when a woman receives relevant and unbiased information about screening, she is able to make an informed decision on breast cancer screening. This happens when she understands and interprets the information received (Davis et al 2010: 174). As can be seen from Chart 11, the media has been the utmost source of information for participants, more than healthcare professionals.

\section{Knowledge on how to do breast self-examination}

One hundred and ten participants answered this question $(\mathrm{N}=110)$. As indicated in Chart 4, sixty participants knew how to do BSE ( $n=60$, $54.55 \%$ ) while 50 participants did not know how to do BSE. According to Petty and Krosnick (2014: 248), the maintenance of strong attitudes is highly dependent on knowledge. The processing of information acquired is facilitated by knowledge. The findings in Chart 12 show that more than $50 \%$ of the respondents had prior knowledge on how to do breast self-examination.

\section{Ways of doing breast self-examination}

Participants were asked to tick as many ways they know to do BSE. As shown in Chart 13, sixty seven participants responded to this question $(\mathrm{N}=67)$. Of the sixty seven participants $(\mathrm{N}=67)$ that responded to question 14 , thirty two knew of only one way of doing BSE $(n=32$, $47.6 \%$ ). Fifteen knew two ways ( $n=15,22.39 \%)$, also, fifteen knew about three ways ( $n=15,22.39 \%)$ and only 5 knew all the four ways of doing BSE $(n=5,7.46 \%)$. This is shown in Chart 14 .

\section{Looking in the mirror}

There were thirty responses that looking into the mirror to feel for any abnormality in the breast is a way to do BSE ( $n=30,44.78 \%$ of 67$)$. The main components of BSE are inspection and palpation. Inspection is done by standing in front of the mirror. Looking into the mirror and using the pads of the fingers to feel for abnormalities is one of the ways to do BSE. Most of the participants were not really familiar with using this method as shown by the results from Chart 13 .

\section{Lying down}

Feeling the breast for abnormalities while lying down had fifty responses ( $n=50,74.63 \%$ of 67$)$. The flat pads of the fingers are used to palpate the breast at different pressure levels and in a specific pattern. This is easily done when lying down in a flat or side-lying partially position. Lying down to examine the breast evenly spreads out the breast tissue along the chest wall. This was the method that most of the participants were familiar with.

\section{In the shower}

There were 28 responses for feeling the breast for abnormalities whiles in the shower ( $n=28,41.79 \%$ of 67$)$. According to Breast Cancer. Org, many women are able to feel their breast tissue well when the skin is wet. The breast is palpated with the pads of the fingers. The entire

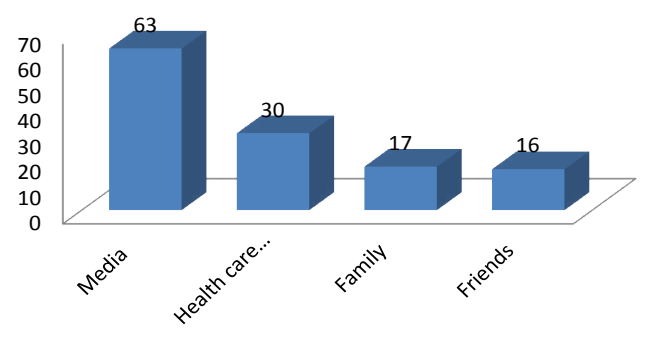

Chart 11: Source of information.

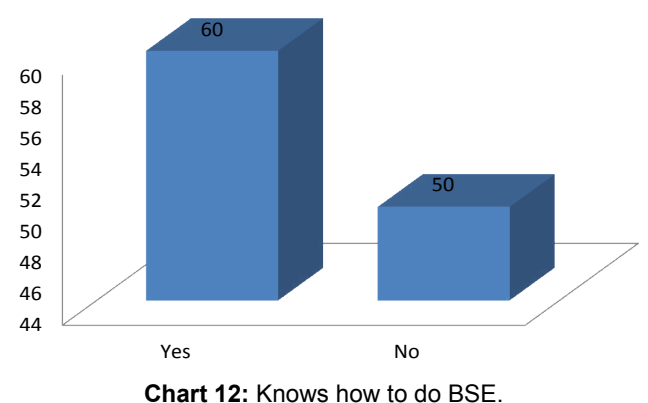

Chart 12: Knows how to do BSE.

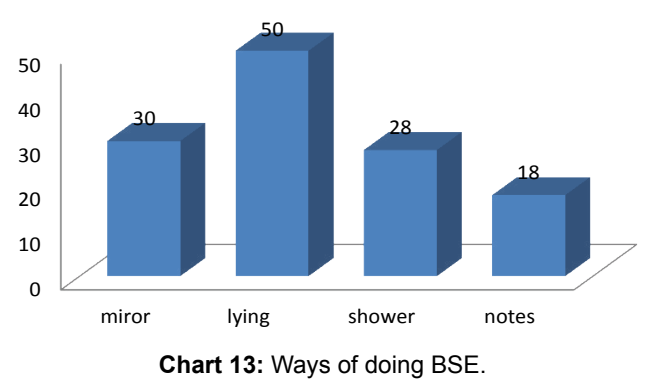




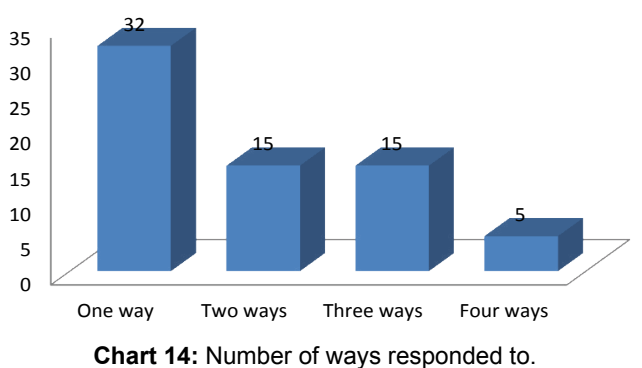

breast is palpated from top to bottom and from side to side. However, this was not the case with participants, as most of them were not familiar with this way of doing BSE.

\section{Making notes}

Eighteen responses were given for making notes of abnormalities found ( $\mathrm{n}=18,26.87 \%$ of 67$)$. After BSE has been done, any abnormality detected should be reported at the hospital for investigations to be done and possible treatment of the abnormality detected. Very few participants were conscious that any abnormality detected must be noted.

\section{Qualitative Data}

Themes

All interviews were transcribed. The themes and sub-themes identified from the interview data are as follows:

Knowledge

- Knowledge on breast cancer.

- Knowledge on breast cancer screening.

- Knowledge on BSE

\section{Knowledge}

The level of knowledge participants had on breast cancer; breast cancer screening and BSE emerged as a theme from interview data. The participants' level of knowledge emerged under the following subthemes.

\section{Knowledge on breast cancer}

A belief or approval condition must be satisfied in order to gain knowledge. This is according to the traditional conception of knowledge. All the participants had knowledge of breast cancer being an existing disease and mostly affect women.

Hmm, breast cancer (inhales) well, it's a disease I'd say, that doesn't mainly affect women, I think according to my understanding, can affect both men and women, but I think it's more common in women. Yes it's just like any other form of cancer I'd say; only that it happens in the breast (P1/Int. 1).

Ehh its cancer that is located in the breast basically, in your mammary gland, I don't know what else to say (P12/ Int. 12).

This is consistent with the responses from the questionnaire where majority of the participants had heard of breast cancer.

Almost all the participants (ten) did not have any prior knowledge on the risk factors of breast cancer. Only two participants had prior knowledge on the risk factors of breast cancer.
I don't know, I just see people with breast cancer, the causes and everything I don't know (P4/Int. 4).

Huh (sighs), let's just say I'm really clueless, I've never done research on it. I don't know, will be lying to you if I say I do (P6/ Int. 6).

However, five participants knew about myths that were supposedly known to be risk factors of breast cancer.

Umm, what comes to mind, causes of breast cancer, umm I think deodorant, antiperspirants. I don't know if the clothing you wear, such as bras, to my understanding something that's repeatedly hurting, umm, I think that's the only ones I know that can cause breast cancer (P10/Int. 10).

I don't know if it's a real cause or it's a myth, like what people say, incorrect bra I think, maybe if it's too tight or moisture, just not taking care of your breast (P7/Int.7).

There are myths in the media and internet about some factors that are thought to be causes of breast cancer. These factors include the use of antiperspirants, underwear bras and wearing bras to sleep in the night. There is no scientific evidence to support these arguments and myths. All perspiration in the armpit cannot be blocked by even the most powerful antiperspirants. The kidneys and liver are responsible for removing toxins from the body, sweating is not the body's significant way of releasing toxins. Also bras do not cause breast cancer. The only scientific study that examined the link between wearing a bra and breast cancer risk did not find any significant difference in the risk between women who wore bras and those who did not (BreastCancer. Org, 2015, Cancer Research UK, 2014).

Majority of the participants did not know the risk factors of breast cancer, as compared to the responses from the questionnaire, most of the participants only had knowledge of family history being a risk factor and did not know other risk factors.

Almost all the participants (eleven) knew that an abnormal lump is a symptom of breast cancer.

Umm, the lump in your breast that's all I know (P11, Int.11).

Most of them (seven participants) did not have any prior knowledge on other signs and symptoms of breast cancer. Four had prior knowledge of pains in the breast being a symptom of breast cancer as did only one having knowledge about other symptoms. Int. 9)

Just the pain and the lump in the breast, besides that, nothing (P9/

I think the colour of your breast and areolar changes, pains in the nipples, umm maybe discharge, the lumps, I have no idea how you determine what the lump are or whether it's a fat gland, because, I feel my breast very lumpy but it's the normal. So I don't know how they figure out what is the normal. I think if the lump is not only in your breast but, in the surrounding areas, sometimes in your armpit. Hmm, I think that's the things that I know of (P10/Int.10).

This is consistent with the findings from the questionnaire where most of the participants did not have prior knowledge on other symptoms of breast cancer other than swelling of the breast and lumps in the breast and armpit.

\section{Knowledge on breast cancer screening}

Almost all the participants (eleven) did not have any prior knowledge on breast cancer screening. 
Hmm, I actually have no knowledge of it. Wow, I don't have any knowledge on it; yeah I can't even say what it is actually. What is breast cancer screening? Can you explain it to me? (Giggles) (P2/Int.2)

Umm, I've never heard of it. I don't know anything about it (P5/ Int.5)

Only one participant had prior knowledge on breast cancer screening and she was not even sure what screening really entailed.

I've heard about it, I think that's when ladies would go for mammograms, is it? (sighs), I've heard about it but I don't think I know too much about it, I just know women would go and request for that kind of examination and it's done but exactly what it entails of, I'm not so sure (P1/Int.1).

The immediate aim of breast cancer screening is to detect cancers before they become evident clinically (IARC, 2002:4, 8). The American Cancer Society and the International Agency for Research on Cancer (IARC) have the following recommendations for breast cancer screening. They recommend that women who are 40 years and above, should have a mammogram every year. This must continue as long as the health of the woman is good. Also for women who are between age 20 and 30, should have a clinical breast examination every 3 years and women at 40 and above every year. Breast self-examination is the recommended option for breast cancer screening in women who are below 30 years of age.

The result does not correspond with that of the questionnaire where majority of the participants were aware of breast cancer screening.

\section{Knowledge on breast self-examination}

Half of the participants (six) had prior knowledge on BSE; they had heard about it but did not know the correct way to examine their breast.

I just know like, you use your hand to examine your own breast and see if you don't have any lumps in, maybe there's a lump or not, from that you'll know okay I have a problem so I should get myself checked. I just touch like the whole breast, I'm not sure how, but I should be of knowledge if something is wrong though (P8/Int.8).

Umm it's actually when you're feeling your breast if there's a lump or something. Just examining if there's a lump in it. Like I just feel my breast if there's any lump or something, just around it (P11/Int.11). BSE.

Only two participants had prior knowledge on how to correctly do

Basically I stand in front of the mirror with my hands down, then I have a look at my breast to check to see which one is bigger, how much bigger, how does it feel and then I lift my arms up and umm (pause) well I check the way it feels, so that the next time I check I know what it looks like and its colour and so forth. I would lift my one arm up and then with the other arm you feel for any bumps other than the usual lumpy feeling, and you feel if it hurts anywhere and what kind of pain and if its associated with any hormonal changes, for example, which part of cycle I'm in, umm and then I do the same on the opposite side and (pause) that's about it (P10/In.t 10)

Four of the participants did not have any prior knowledge on BSE.

No, I haven't heard of it before, I won't lie. No I don't know what it is. I don't know (P3/Int.3).

This is consistent with the findings from the questionnaire where majority of the participants had heard of breast self-examination but, very few knew the correct ways of doing BSE.

\section{Recommendations}

Education on breast cancer and cancer as a whole should be initiated in high schools and higher institutions of learning as part of their curriculum.

Posters on breast cancer screening and BSE should be put up at public places and campuses.

Information sheets and leaflets on breast cancer should be made available at the library, in the post card stands in the residences of higher institutions and health centres and clinics where women can easily have access to them.

Health education and promotion must be done by oncology specialist in schools, communities and places of public gatherings.

Primary health care providers should provide education on breast cancer and BSE in their clinics.

Health care professionals should do more awareness in rural areas and schools on breast cancer

Posters showing how to do BSE should be put up in public places and campuses

Breast awareness campaigns must be done every month, not only in October which is the breast cancer awareness month.

Health care professionals should give information on breast cancer to women when they visit the hospital or health centre.

\section{Recommendations to Policy makers}

- Introduction of cancer education in the curriculum of the education system.

- Training of community health nurses on tailored health education to specific target groups.

- Training of volunteers as health promoters for breast cancer screening and BSE.

- Hospitals and health centres should give information to women on breast cancer screening.

- The mobile clinics should not only be giving counselling and testing for HIV but should also include clinical breast examination and giving information on breast cancer.

- Posters and information leaflets on breast cancer screening and BSE should be available at all public places and campuses

- Policies on women's health should be reinforced by policy makers.

\section{Conclusion}

The aim and objectives that this study had set out to do, have all been achieved. The study highlighted the significance of educating females on the importance of BSE. The importance of females having the correct attitude, as well as knowledge on breast cancer, in order to practice BSE on a regular basis also came to light.

Finally, this study emphasised how vital it is for all health professionals to keep updated with new knowledge and technology in order to do health promotion and give advice in this regard to females. This is particularly important for Registered Nurses at primary health care level, as they are the first point of contact with all clients. 
Citation: Ansah MB, Vember H (2018) Female Students Knowledge of Breast Self-examination in a University in the Western Cape. J Oncol Med \& Pract 1: 115. doi:10.4172/2576-3857.1000115

Page 8 of 8

The researcher hopes that further research, will be undertaken, particularly in rural areas, in order to educate more females with regard to BSE. It is also the wish of the researcher that, policy makers, particularly in the education and health sector, should include BSE and other cancer awareness programs in their curricula from primary to secondary school levels.

\section{References}

1. Timothy S, Curtis EM, Tabar (2012) Intraocular medulloepithelioma. Arch Pathol Lab Med 136: 216-212.

2. Mojgan D (2014) Medulloepithelioma of the ciliary body. Bull French Div AIP 60: 159-164.

3. Reese AB (1957) Medulloepithelioma (dictyoma) of the optic nerve. Am J Ophthalmol 44: 4-6.

4. Green WR, lliff WJ, Trotter RR (1974) Malignant teratoid medulloepithelioma of the optic nerve. Arch Ophthalmol 91: 451.

5. Anderson SR (1962) Medulloepithelioma of the retina. Int Ophthalmol Clin 2: 483-506.
6. Mullaney J (1974) Primary malignant medulloepithelioma of the retinal stalk. Am J Ophthalmol 77: 499-504.

7. Saunders T, Margo CE (2012) Intraocular medulloepithelioma. Arch Patho Lab Med. 136: 212-216.

8. Lloyd III WC, O'Hara M (2001) Malignant teratoid medulloepithelioma: Clinicalechographic-histopathologic correlation. J AAPOS 5: 395-397.

9. Zimmerman LE (1970) The remarkable polymorphism of tumors of the ciliary epithelium. Part 1. Trans Austr Coli Ophthalmol 2: 114-125.

10. Andersen $S$ (1971) Differentiation features in some retina tumor and in dysplastic retinal conditions. Am J Ophtalmol 1: 231-241.

11. Kramer GD, Arepalli S, Shields CL, Shield JA (2014) Ciliary body medulloepithelioma association with pleuropulmonary blastoma in familial tumour predisposition syndrome. J Pediatr Ophtalmol Strabismus 51: e48-50.

12. Priest JR, Williams GM, Manera R, Jekinson H, Brûndler MA, et al. (2011) Ciliary body medulloepithelioma: Four cases associated with pleuropulmonary blastoma: A report from the International pleuropulmonary blastoma registry. Br J OPhtalmol 95: 1001-1005.

13. Yanko L, Behar A (1978) Teratoid intraocular medulloepithelioma. Am J Ophtalmol 85: 850-853. 